\title{
The unspoken benefit of participation in a clinical trial
}

\author{
Authors: Konstantin Schwarz, ${ }^{\mathrm{A}}$ Sathish Parasuraman, ${ }^{\mathrm{B}}$ Satnam Singh, ${ }^{\mathrm{C}}$ John D Horowitz, ${ }^{\mathrm{D}}$ Dana K Dawson ${ }^{\mathrm{E}}$ \\ and Michael P Frenneaux ${ }^{\mathrm{F}}$
}

\section{Background}

Publicly funded trials do not usually offer financial incentives to volunteers. An intensive level of medical care could act as an additional motivator for participation. Our aim was to establish whether patients may draw any clinical benefit from volunteering in a clinical trial.

\section{Methods}

We analysed the recruitment process of a phase II randomised controlled trial, the Inorganic Nitrate in Angina Study.

\section{Results}

Two-hundred and thirteen patients with a history of stable angina and who had been under at least annual primary care review were screened for participation by history taking, examination, 12-lead electrocardiography, treadmill test and echocardiography. Thirty-five ( $16.4 \%)$ patients were found to have significant unstable or new clinical pathology, requiring urgent clinical attention. We identified 17 (7.9\%) patients with unstable angina. Furthermore, we found new undiagnosed pathologies: amyloidosis in two (0.9\%), hypertrophic cardiomyopathy in two $(0.9 \%)$, left ventricular systolic dysfunction (ejection fraction $<45 \%$ ) in three $(1.4 \%)$, left ventricular thrombus in one $(0.4 \%)$, significant valvular disease in five $(2.4 \%)$ and arrhythmias in six $(2.8 \%)$.

\section{Conclusion}

Compared with routine care, patients screened for a clinical trial may come under an increased level of scrutiny that may affect their clinical management. This may act as additional motivator to attract patients to future studies.

KEYWORDS: clinical research, recruitment

DOI: 10.7861/clinmed.2021-0292

Authors: ${ }^{\text {A }}$ consultant cardiologist, Karl Landsteiner University of Health Sciences, Krems, Austria; ${ }^{B}$ Consultant cardiologist,

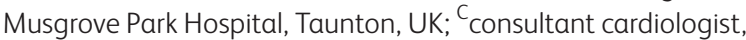
Frimley Park Hospital, Frimley, UK; D professor of cardiovascular medicine, Adelaide Medical School, Adelaide, Australia; ${ }^{\text {p }}$ rofessor of cardiovascular medicine, University of Aberdeen School of Medicine and Dentistry, Aberdeen, UK; F professor of cardiovascular medicine, Norwich Medical School, Norwich, UK and Academic Health System Hamad Medical Corporation, Doha, Qatar

\section{Introduction}

Patients who take part in clinical trials frequently volunteer a significant amount of their free time, undergoing intense testing and interventions as part of their participation. Most publicly funded clinical trials do not make provision for financial incentives to volunteering patients, apart from covering their travelling and catering costs. According to the Handbook for good clinical research practice, reimbursement is possible, however, should not be so large as to unduly induce subjects to enrol in a study or stay in the study when they would otherwise withdraw. ${ }^{1}$

Recruitment is, therefore, based on patients' altruism in order to develop new diagnostic or treatment strategies and potentially help future patients. Nevertheless, it is possible that some volunteers may benefit from their close clinical assessment and care, while undergoing the rigorous screening process to be included in a clinical trial. Here, we analysed any potential effects of screening patients with a history of angina for a previously published trial. These patients had been under regular medical review prior to screening.

\section{Methods}

The Inorganic Nitrate in Angina Study (INAS) was a phase II parallel design randomised double-blind controlled trial investigating the potential benefit of inorganic nitrate supplementation on the exercise capacity of patients with stable angina. The study was conducted according to the Declaration of Helsinki, approved by Scotland A Research Ethics Committee (SAREC) and subject to Medicine and Healthcare products Regulatory Agency (MHRA) regulations.

The detailed study protocol and results were previously published elsewhere. ${ }^{2,3}$ In brief, all participants had a previously established diagnosis of stable angina, and were screened for participation with history taking, clinical examination and 12-lead electrocardiography (ECG). Thereafter, they underwent a series of baseline ECG treadmill tests (ETT) and resting transthoracic echocardiography. In patients with positive ETT, who did not have angiographic evidence of recent obstructive coronary artery disease, an additional positive non-invasive test (dobutamine stress echocardiography or myocardial perfusion scan) was required prior to treatment arm randomisation. Those patients in whom unexpected pathologies were identified, or in whom the angina was considered to be unstable, were excluded from the investigational part of the trial (prior to randomisation). Appropriate further investigation and treatment or referral was arranged. The reasons for exclusion were documented 
and analysed in this study and expressed numerically and as a frequency distribution. Our participants did not receive financial incentives, however, they were offered travel compensation (usually arranging a taxi or parking expenses) and refreshments on days when they spent several hours in our trial research office.

\section{Results}

In total, over 1,600 patients from primary and tertiary care with a diagnosis of angina or ischaemic heart disease were invited to participate. These patients were approached while attending a routine cardiology outpatient visit or following a previous positive coronary angiography result at Aberdeen Royal Infirmary. Following initial clinical history and review of inclusion and exclusion criteria, 520 patients from the cardiology department were invited to participate (another 2,896 patients from an angiographic list database were screened but not found eligible). Similarly, 1,119 potentially suitable patients were invited by post from thirteen collaborating general practice (GP) surgeries across Aberdeenshire using their own local databases information (where the main screening criterion was active glyceryl trinitrate prescription and a history of either angina or ischaemic heart disease). All responders underwent a telephone interview to establish whether there were current exertional anginal symptoms and any potential exclusion criteria (eg ability to walk on treadmill) before invitation for a personal screening visit to the research facility. Two-hundred and thirteen such preselected patients were consented and screened in the department. Out of all consented patients, 138 (64\%) came from GP sources, 69 (32.4\%) from cardiology and six (2.8\%) from other sources. Other sources included recruitment posters in public places (hospital, pharmacy and supermarkets) and personal promotion at cardiovascular exercise classes (frequented by former cardiac rehabilitation patients).

Following two baseline screening visits, 70 (out of 213) patients were found eligible and were randomised to one of the two treatment sequences (placebo-nitrate or nitrate-placebo). The main reasons for exclusion in patients screened in the research facility were $<1 \mathrm{~mm}$ ST depression on ETT in 108 (76\%), failure to reproduce $1 \mathrm{~mm}$ ST depression within 15\% time difference on two separate occasions or no evidence of reversible ischaemia on alternative non-invasive test $10(7 \%)$, left bundle branch block in six $(4 \%)$, resting ST depression $>1 \mathrm{~mm}$ in three $(2 \%)$ and other reasons in $16(11 \%)$

Several patients had more than one reason for exclusion, and indeed needed further medical attention. We identified 17 (7.9\%) patients with unstable angina, all of which were referred for urgent outpatient coronary angiography. Three of these later underwent coronary artery bypass surgery, two patients were treated with coronary angioplasty, eight patients had alteration of their pharmacological management and one patient had no change in treatment. Unfortunately for three patients, no follow-up information on their clinical outcome was available. We also made the several new diagnoses: amyloidosis in two $(0.9 \%)$, hypertrophic cardiomyopathy in two $(0.9 \%)$, previously unknown impaired systolic function with ejection fraction $<45 \%$ ) in three $(1.4 \%)$, left ventricular thrombus in one $(0.4 \%)$, atrial fibrillation/ flutter in four (1.8\%), high burden of ventricular extrasystoles in one $(0.4 \%)$, bifascicular block in one $(0.4 \%)$ and significant valvular abnormality in five $(2.3 \%)$, of which there was moderate to severe aortic stenosis in two $(0.9 \%)$ and severe mitral regurgitation in three $(1.4 \%)$

\section{Discussion}

Following the atrocities of World War II, at the 1947 International Medical Tribunal in Nuremberg, the court articulated a 10-point set of rules for the conduct of human experiments, the so-called Nuremberg Code. ${ }^{4}$ According to this, no persuasion or pressure of any kind should be put on clinical trial participants.

Monetary compensation is sometimes used to incentivise recruitment of trial or survey participants who might otherwise not take part. ${ }^{5}$ Financial incentives are often critically viewed by ethical review boards.

In telephone surveys, monetary incentives were often shown to be more effective than non-financial incentives. ${ }^{6}$ Singer and Bossarte, and Wertheim and Miller discussed whether modest financial incentives can be coercive. ${ }^{7,8}$ Both came to the conclusion that monetary offers may exert undue influences but cannot be coercive. Participation in research has been previously analysed widely on a cost-benefit basis. ${ }^{5}$ Dunn and Gordon have argued that, since economic forces operate in any case, researchers must take them into account. ${ }^{9}$ When should financial incentives be considered unduly influential? Singer and Couper proposed a rule that undue influence is exerted if financial incentives result in participants undertaking risks which they would not be willing to accept without the incentive. ${ }^{5}$ Recently, Vellinga et al presented a discrete choice experiment (DCE) to explore preferences and the use of different incentive methods for clinical trials. This DCE can be adapted to specific clinical trial scenarios and its results used for ethical applications while identifying the most favourable incentives for participation in clinical trials. ${ }^{10}$

Whereas financial compensation for clinical trial participants has often raised ethical questions in regard to its influence on the integrity of the research, other potential benefits of participation are frequently quoted in patient information sheets to motivate patients. ${ }^{11,12}$ Among these are access to new promising treatments often not available outside of the trial setting; close monitoring, advice and care by the research team; the chance to get greater understanding of the patients' condition; the chance to help society and future patients; and adding to scientific knowledge.

Although there are limited data available on clinical benefit by participation to specific individual studies, there are previous trials documenting better clinical patient outcomes in hospitals that are participating in clinical trials compared with hospitals not enrolling their patients into research. ${ }^{13,14}$ It has been speculated that the reasons for this observation could be that trial investigators are frequently opinion leaders with access to new drugs and devices, and frequently adhere to the most up-to-date optimal medical treatment guidelines. Other reasons may include the availability of expensive modern treatment options that are available in financially better equipped and often larger trial centres. It has been suggested that patients participating in trials benefit from superior treatment by adhering to improved clinical care within a trial (the Hawthorne Effect). ${ }^{15}$ None of our patient volunteers who came for screening received any financial incentives for their participation, however, many benefited indirectly from a thorough medical examination. As a 'side effect' of this widespread screening, we diagnosed a number of pathologies or undiagnosed severe progression of their underlying conditions, usually making these patients non-eligible for our study. This was quite surprising as all these patients were under regular medical care for their angina. Sixteen per cent of 
our patients needed further clinical care. This is quite high and will certainly be related to the carefully selected population screened for the INAS trial and the local provision of clinical care providers. The specifics of the study protocol may limit the translation of its numerical results to other populations at different times.

Some of our results may be considered to reflect negatively on the provision of standard care, however, this simplistic conclusion is contentious in view of the preselected patient population that we were looking for. Nearly half of our unheralded diagnoses were due to unstable angina. In most cases, this was due to very recent significant symptom worsening of their previously known stable angina (development of new Canadian Cardiovascular Society class III or IV angina). This can occur very quickly within days or weeks and regular yearly follow-up may not be best suited to pick up such unexpected events. Similarly, as most patients were under regular primary care follow-up, rather than specialist care, certain diagnoses requiring access to echocardiography (left venricular thrombus or severe valvular pathology) may have been very difficult to establish by clinical examination only. We would rather highlight our excellent experience in collaboration with the local primary and secondary care centres and reflect on the enhanced clinical care offered by involvement in our clinical trial.

It has to be acknowledged that volunteering for additional medical tests during research screening can potentially lead to harm and unnecessary downstream investigations. During our screening tests, none of our volunteers experienced any serious harm, however, two of our patients experienced short supraventricular tachycardias during higher stages of dobutamine stress echocardiography (DSE). These tachyarrhythmias had to be reverted by intravenous beta-blocker bolus injection. It is difficult to quantify the impact of potentially unnecessary downstream investigations that happened to our patients once referred back to their usual clinical care providers for further management of our newly diagnosed pathologies. Nevertheless, in light of some highly concerning newly found pathologies (left ventricular thrombus, significant valvular pathologies or untreated arrhythmias), we speculate that the impact of targeted management far outweighs the risk of few potentially unnecessary downstream investigations. The decision whether and how our clinical colleagues further managed our screened patients was left entirely in their hands. We hope that principles of evidence-based medicine and justification balancing benefit vs harm would have been followed.

Surprisingly, despite the frequent inclusion in patient information sheets of a statement that patients 'may benefit from closer monitoring and care from the research team', to the best of our knowledge, no previous trials have published any data analysing the real frequency of clinical impact on patients who were screened but then not recruited to clinical trials.

Our data might contribute in future to substantiate the claim that patients may benefit from participation in clinical studies, independent of the intended experimental treatment.

\section{Acknowledgements}

We are grateful to Valerie Harries for assisting with data collection.

\section{Funding}

The study was funded by the Medical Research Council.

\section{References}

1 World Health Organization. Handbook for good clinical research practice. WHO, 2002. www.who.int/medicines/areas/quality safety/safety_efficacy/gcp1.pdf

2 Schwarz K, Singh S, Parasuraman SK et al. A randomized doubleblind placebo-controlled crossover trial of sodium nitrate in patients with stable angina INAS. Future Cardiol 2016;12:617-26.

3 Schwarz K, Singh S, Parasuraman SK et al. Inorganic Nitrate in Angina Study: A Randomized Double-Blind Placebo-Controlled Trial. J Am Heart Assoc 2017;6:e006478.

4 Moreno JD, Schmidt U, Joffe S. The Nuremberg Code 70 years later. JAMA 2017:318:795-6.

5 Singer E, Couper MP. Do incentives exert undue influence on survey participation? Experimental evidence. J Empir Res Hum Res Ethics 2008:3:49-56.

6 Singer E, Van Hoewyk J, Gebler N, McGonagle K. The effect of incentives on response rates in interviewer-mediated surveys. Journal of Official Statistics 1999:15:217-30.

7 Singer $E$ and Bossarte RM. Incentives for survey participation: when are they 'coercive'? Am J Prev Med 2006;31:411-8.

8 Wertheimer A, Miller FG. Payment for research participation: a coercive offer? J Med Ethics 2008;34:389-92.

9 Dunn LB, Gordon NE. Improving informed consent and enhancing recruitment for research by understanding economic behavior. JAMA 2005;293:609-12.

10 Vellinga A, Devine C, Ho MY et al. What do patients value as incentives for participation in clinical trials? A pilot discrete choice experiment. Research Ethics 2020;16:1-12.

11 Pandya M, Desai C. Compensation in clinical research: The debate continues. Perspect Clin Res 2013;4:70-4.

12 Dickert N, Grady C. What's the price of a research subject? Approaches to payment for research participation. N Engl J Med 1999;341:198-203.

13 Majumdar SR, Roe MT, Peterson ED et al. Better outcomes for patients treated at hospitals that participate in clinical trials. Arch Intern Med 2008;168:657-62.

14 Boaz A, Hanney S, Jones T, Soper B. Does the engagement of clinicians and organisations in research improve healthcare performance: a three-stage review. BMJ Open 2015;5:e009415.

15 Braunholtz DA, Edwards SJ, Lilford RJ. Are randomized clinical trials good for us (in the short term)? Evidence for a' trial effect'. J Clin Epidemiol 2001;54:217-24.

Address for correspondence: Dr Konstantin Schwarz, Department of Internal Medicine 3, University Hospital Sankt Pölten, Dunant-Platz 1, 3100 Sankt Pölten, Austria. Email: konstantin.schwarz@stpoelten.lknoe.at Twitter: @konstantin1000; @drsathish82; @Satnam_Cardio; @dana_dawson16 